\title{
P04-2-32 Poster session
}

\section{WNK1 is required for the proliferation induced by hypotonic challenge in vascular cells}

\author{
Yong-Yuan Guan, Ya-Juan Zhang \\ Pharmacology, Zhongshan School of Medicine, Sun Yet-Sen University, China
}

Background:Hypotonic challenge evoked vascular cell proliferation through activation of volume-regulated Cl-channel (VRCC), leading to the decline of intracellular $\mathrm{Cl}$-concentration ([Cl- $] \mathrm{i})$.We suppose that the decrease of [Cl-]i may active one or more $\mathrm{Cl}$-sensitive kinases,resulting in subsequent signal cascade. We investigated whether WNK1 involves in VRCC-induced proliferative signal pathway in vascular cells

Methods:Bromodeoxyuridine(BrdU)incorporation and flow cytometry were used for proliferation determination. Western blot and cDNA/siRNA transfection were used for signal pathway determination in cultured A 10 vascular Cells.

Results:Hypotonic challenge induced WNK1 phosphorylation without any alteration of WNK1 protein expression. Hypotonic-induced A10 cell proliferation was significantly increased by over expression of WNK1, and inhibited by silence of WNK1.WNK1 mutation did not affect phosphorylation and cell proliferation.Silence of WNK1 caused cell cycle arrest in G0/G1 phase and prevented the entrance from G1 to S phase, whereas,over expression of WNK1 accelerated cell cycle transition from G1 to S phase.Silence of WNK1 inhibited cyclin D1/cyclin E1 expression,and increased P27KIP/P21CIP expression. Whereas, overexpression of WNK1 increased cyclin D1/cyclin E1 expression,and reduced P27KIP/P21CIP expression.Furthermore,WNK1 knockdown or overexpression significantly attenuated or increased phosphorylations of Akt and PI3K induced by hypotonic challenge,respectively.

Conclusion:The reduce in [Cl-]i by hypotonic challenge-indced VRCC opening evokes WNK1 phosphorylation, which mediates cell cycle transition from G0/G1 to S phase and proliferation through PI3K-Akt signal pathway in A10 VSMCs. 